\title{
Milieu-Initiated Inversion of the Aqueous Polyproline II/Beta Propensity in the Alanine Tripeptide: Energetics Origin of the Onset of Amyloid Formation
}

Noemi G. Mirkin and Samuel Krimm*

LSA Biophysics, University of Michigan

930 N. University Ave., Ann Arbor, MI 48109

* Phone: 734 763-8081

Email: skrimm@umich.edu

Fax: 734 764-3323 


\begin{abstract}
Extending our earlier analogous study of the alanine dipeptide (ADP), we have now analyzed the effect of the external environment on the polyproline II (P) and $\beta$ relative energies, the $\mathrm{P} / \beta$ propensity, of the alanine tripeptide (ATP). Ab initio calculations of $\operatorname{ATP}\left(\mathrm{H}_{2} \mathrm{O}\right)_{19}$ and $\mathrm{ATP}\left(\mathrm{H}_{2} \mathrm{O}\right)_{19}(\mathrm{HCl})$ exhibit the same propensity inversion as in ADP: in the pure-water case the PP conformation is favored while the addition of the $\mathrm{HCl}$ molecule results in the $\beta \beta$ conformation being of lower energy. A comparison, following an intermediate insertion and departure of an $\mathrm{HCl}$ molecule, shows that the energy of a hydrogen-bonded $\left(\mathrm{H}_{2} \mathrm{O}\right)_{19} \beta$ ATP:: $\beta \mathrm{ATP}\left(\mathrm{H}_{2} \mathrm{O}\right)_{19}$ structure is lower than that of the sum of two separate PP systems, i.e., that the aggregated state of the peptide is favored. This arises from the basic physical response to their total environmental influences. Questions about quantitative results from molecular dynamics simulations, obviously needed to analyze longer chains and other side chains, are addressed via rigid-water calculations. The desirability of basing studies of amyloid formation on our proposed alternative milieufolding paradigm is discussed.
\end{abstract}




\section{INTRODUCTION}

It is now generally accepted, as proposed ${ }^{1}$ and substantiated ${ }^{2}$, that a significant contribution to the circular dichroism (CD) spectra of unordered polypeptides and proteins in aqueous solution (a strong negative band near $200 \mathrm{~nm}$ and a weak positive band near $220 \mathrm{~nm}$ ) comes from locally ordered chain conformations close to that of the three-fold left-handed helix of polyproline II (P). Our ab initio studies of a hydrated alanine dipeptide (ADP) indicated that this preference over that of the nearby $\beta$ conformation is determined primarily by the difference in the interaction energies associated with their peptide-water and water-water hydrogen-bonded structures ${ }^{3}$. The idea that the relative $\mathrm{P}$ and $\beta$ energies (i.e. $\mathrm{P} / \beta$ propensity) depend on the specific features of these structures motivated a detailed study of the possible influence on this propensity of other species in the aqueous environment and resulted in our analogous investigation of this property of hydrated $\mathrm{ADP}$ with an added $\mathrm{HCl}$ molecule. Our finding was that the $\mathrm{P} / \beta$ propensity becomes inverted, that is the energy of the $\beta$ conformer is now lower than that of the $\mathrm{P}$ conformer ${ }^{4}$.

The recognition that this could have implications for the formation of plaques in amyloidosis diseases was further supported by showing that a hydrated hydrogen-bonded two- $\beta$-peptide state is energetically favored over one in which the two initial hydrated $\mathrm{P}$ components are separate, an indication that aggregation of $\beta$ chains is to be expected in favorable environments. Although this emphasized the importance of accepting a peptide-medium paradigm as the basis of structure determination, it was also clear that further detailed study of peptides was needed. Aside from the likelihood that other environmental conditions besides $\mathrm{pH}$ could initiate such changes, it would be necessary 
to explore the influence of factors such as chain length and side-chain composition. As a start in this direction, we report here on an ab initio study of the $\mathrm{P} / \beta$ propensity in the hydrated alanine tripeptide (ATP) in the absence and presence of an $\mathrm{HCl}$ molecule. We also consider the important issue of reproducing by molecular dynamics the accuracy of such basic quantum-mechanical calculations in needed studies of longer chains and other side chains, and deal as well with the implications of our results for achieving a deeper understanding of amyloid formation.

\section{CALCULATIONAL METHODS}

The ATP molecule, $\mathrm{CH}_{3}-\mathrm{CONH}-\mathrm{C}_{1} \mathrm{H}\left(\mathrm{CH}_{3}\right)-\mathrm{CONH}-\mathrm{C}_{2} \mathrm{H}\left(\mathrm{CH}_{3}\right)-\mathrm{CONH}-\mathrm{CH}_{3}$, hydrated with water was minimized in the following way: 1) the peptide was constructed with the canonical values of $\varphi, \psi\left(\mathrm{P}:-75^{\circ}, 145^{\circ}\right.$ and $\beta$ : $\left.\left.-134^{\circ}, 145^{\circ}\right) ; 2\right)$ first-layer waters were added to the ubiquitous peptide hydrogen-bonding positions, i.e., two to the $\mathrm{CO}$ and one to the $\mathrm{NH}$, comprising a total of 9 water molecules, and the entire system was minimized; 3) using the new $\varphi, \psi$ as a starting point and adding sufficient waters to complete the second layer, 10 in this case for a total of 19 , the system was again totally relaxed and minimized. As before ${ }^{4}$, the global and some local minima of each conformation were determined. For the $\operatorname{ATP}\left(\mathrm{H}_{2} \mathrm{O}\right){ }_{19}(\mathrm{HCl})$ system, as previously ${ }^{4}$, the $\mathrm{HCl}$ was added to the above minimized structures in a variety of ways: as an intact molecule initially located in numerous positions with respect to the water layers and as initially separate ions in various locations and the total system was minimized. In all cases the final structures had separated ions imbedded in the water environment. 
Calculations were done with Gaussian $09^{5}$, with the dispersion-corrected wB97X-D functional $^{6}$ and the $6-31++\mathrm{G}^{* *}$ basis set. In addition, a Polarized Continuum Model (PCM) reaction field calculation was done on the final minima in order to assess the impact of general polarization contributions from the surrounding medium on the final structures and energies. Atomic charges were determined as Natural Population Analysis (NPA) charges.

\section{RESULTS AND DISCUSSION}

Alanine Tripeptides. The energy difference of importance in the ATP systems is that between the $P_{1} P_{2}$ and $\beta_{1} \beta_{2}$ conformations, i.e., $\Delta E=E\left(P_{1} P_{2}\right)-E\left(\beta_{1} \beta_{2}\right)$. The $P / \beta$ propensity inversion is defined by the sign change of $\Delta \mathrm{E}$. This quantity for $\operatorname{ATP}\left(\mathrm{H}_{2} \mathrm{O}\right)_{19}$ is shown in Table 1 . The $-1.80 \mathrm{kcal} / \mathrm{mol}$ for $\beta_{1} \beta_{2}$ indicates that its energy is higher than that of $\mathrm{P}_{1} \mathrm{P}_{2}$ (remembering that each of the individual energies is negative). This value is in line with the result of $-1.72 \mathrm{kcal} / \mathrm{mol}$ for the comparable $\Delta \mathrm{E}$ of $\mathrm{ADP}\left(\mathrm{H}_{2} \mathrm{O}\right)_{12}{ }^{4}$ in indicating the preferred stability of the $\mathrm{P}_{1} \mathrm{P}_{2}$ conformation in an all-aqueous environment (even though the energy of an isolated $\beta_{1} \beta_{2}$ structure with the $\varphi, \psi$ of the hydrated structure is $1.28 \mathrm{kcal} / \mathrm{mol}$ lower than that of the comparable $\left.\mathrm{P}_{1} \mathrm{P}_{2}\right)$. Such an energy difference is associated with the different total interaction energies (i.e., peptide-water and water-water) in the two structures ${ }^{4}$, part of which shows up as a difference in total charge on the peptide, in this case -2 me on $\mathrm{P}_{1} \mathrm{P}_{2}$ and 37 me on $\beta_{1} \beta_{2}$ (compared to zero for the neutral isolated structures). The inclusion of a PCM reaction field in the calculation does not change the $\varphi, \psi$ appreciably but the $\Delta \mathrm{E}$ increases to $-2.62 \mathrm{kcal} / \mathrm{mol}$ and, presumably as a result of the additional effects of polarization and charge transfer 
through water-peptide hydrogen bonds ${ }^{7,8}$, the relevant peptide charges become more positive, at 28 me and 59 me, respectively.

The situation for $\operatorname{ATP}\left(\mathrm{H}_{2} \mathrm{O}\right)_{19}(\mathrm{HCl})$, Table 1, is significantly different from the $\operatorname{ATP}\left(\mathrm{H}_{2} \mathrm{O}\right)_{19}$ case : as with $\operatorname{ADP}\left(\mathrm{H}_{2} \mathrm{O}\right)_{12}(\mathrm{HCl})$, the $\mathrm{P} / \beta$ propensity is inverted, with the $\beta_{1} \beta_{2}$ conformer now being $2.47 \mathrm{kcal} / \mathrm{mol}$ more stable than $\mathrm{P}_{1} \mathrm{P}_{2}$. The peptide charges are significantly more negative, at -30 me and -6 me respectively, but again the $\beta_{1} \beta_{2}$ charge is more positive than that on $\mathrm{P}_{1} \mathrm{P}_{2}$. The inclusion of a PCM reaction field results in a modest increase in $\Delta \mathrm{E}$, to $2.86 \mathrm{kcal} / \mathrm{mol}$, and the peptide charges again become more positive. It is interesting that the positive charge increase from $P_{1} P_{2}$ to $\beta_{1} \beta_{2}$ is accompanied (although not quantitatively) by an increase in the negative charge on the $\mathrm{Cl}^{-}$ion.

The inversion of the $\mathrm{P} / \beta$ propensity in the $\mathrm{HCl}$ case was found to favor peptide aggregation in $\operatorname{ADP}\left(\mathrm{H}_{2} \mathrm{O}\right)_{12}{ }^{4}$ (an inadvertent reversal of the $\mathrm{P}$ and $\beta$ values occurred in the 2.48 and 0 of Table II in reference 4 ), and we examined if the same is true for the tripeptide. The result can be inferred from the following energy comparison. We focus on two individual ATP molecules: 1) in a pure aqueous environment both ATPs are preferably in the $\mathrm{P}_{1} \mathrm{P}_{2}$ conformation, i.e., "solubilized"; 2) an $\mathrm{HCl}$ molecule attaches to one ATP, which then assumes the more favorable $\beta_{1} \beta_{2}$ conformation; to reach the final state we now suppose that the other $\mathrm{P}_{1} \mathrm{P}_{2}$ peptide converts to $\beta \beta$ and it hydrogen-bonds (:::) to the $\beta_{1} \beta_{2}$ peptide to form the ATP:::ATP $\left(\mathrm{H}_{2} \mathrm{O}\right)_{38}(\mathrm{HCl})$ complex, which will lower the energy $\left.{ }^{4} ; 3\right)$ the $\mathrm{HCl}$ molecule departs and we compare the total energy of this system with that of 1 ), the originally separated $\mathrm{P}_{1} \mathrm{P}_{2}$ components. The energy of 3 ) was determined by forming the optimally hydrogen-bonded antiparallel ATP:::PTA pair 
(comparable parallel-chain pairs are also likely for longer chains), adding the necessary water molecules in the initial layers, relaxing the system and minimizing, followed by subsequent additional water layers with relaxation and minimization until a final minimized $\left(\mathrm{H}_{2} \mathrm{O}\right)_{19}\left(\mathrm{ATP}::: \mathrm{PTA}\left(\mathrm{H}_{2} \mathrm{O}\right)_{19}\right.$ structure was achieved. The energy of this structure is $22 \mathrm{kcal} / \mathrm{mol}$ lower than that of 1) (a PCM calculation maintains the sign of this difference), which is not unexpected since there are more non-hydrogen-bonded water $\mathrm{OH}$ groups in 1) than in 3) that are not compensated by the few peptide-peptide hydrogen bonds in 3 ) or by the small energy cost of converting both $\mathrm{P}_{1} \mathrm{P}_{2}$ to $\beta_{1} \beta_{2}$. In the longer chain cases we expect that similar prior constraints on near-peptide water molecules in the $\mathrm{P}$ conformation are released in the $\beta$ conformation thus leaving them to participate in stronger water-water hydrogen-bonding arrangements. In effect, the $\mathrm{HCl}$ has acted like a chaperone-type enabler to the transition from a solubilized system to one in which the peptide components tend to aggregate. If these structures precipitate, removing the peptides from circulation, the system will re-equilibrate and be repeatedly driven toward aggregation, a situation that is extremely unlikely dynamically in 1). Of course, if both molecules in 1) have $\mathrm{HCl}$ entrained and therefore in $\beta \beta$ conformations then the probability of forming an aggregate is enhanced. (The true propensities are obviously determined by the free energies, but if the energy profiles in the ATP cases are similarly shaped, as in the ADP case ${ }^{4}$, implying that the entropies of the two structures are about the same, the free energies will track the enthalpies.) It might be thought that this result is just an abstract theoretical exercise, but there has long been strong x-ray diffraction evidence of its occurrence in low molecular weight synthetic polypeptides, with the formation of cross-beta structures ${ }^{9}$ (in which the local peptide chain axis is 
perpendicular to the global fibril axis), and indeed is a long-known property of proteins ${ }^{10 \text {, }}$

${ }^{11}$. It thus has to be concluded that such aggregation is a natural consequence of originally solubilized peptide conformations in the presence of appropriate milieus.

As noted before ${ }^{4}$, the magnitude of the calculated aqueous $\mathrm{P} / \beta$ propensity inversion could depend on the chain length and the nature of the side chain. Since these properties need to be studied, and obtaining such correlations from the ab initio methods used here is clearly not a viable computational procedure, the necessary route has to be through molecular dynamics (MD) calculations (which in addition can also provide free energies). Given the sensitive dependence of the results on the detailed nature of the various hydrogen-bonding interactions in such systems, it is appropriate to ask if current MD force fields are up to this task, even qualitatively at the level of ab initio calculations. Since charge transfer is not incorporated in present force fields (and, as shown in Table 1, the relative magnitudes could be significant) and even charge flux terms (championed by some to improve accuracy ${ }^{12,13}$ ) remain to be dealt with in current force fields, this is an open question. A simple example of the problem is seen from the results of calculations with rigid water molecules, a common assumption in many present MD simulations ${ }^{14}$. For $\operatorname{ATP}\left(\mathrm{H}_{2} \mathrm{O}\right)_{19}$, although the $\varphi, \psi$ are almost identical to the flexible water case, $\Delta \mathrm{E}=-$ $3.86 \mathrm{kcal} / \mathrm{mol}$ for the rigid water case compared to the $-1.80 \mathrm{kcal} / \mathrm{mol}$ for flexible water (with PCM, again with almost identical $\varphi, \psi$, the comparable values are $-6.55 \mathrm{kcal} / \mathrm{mol}$ and $-2.62 \mathrm{kcal} / \mathrm{mol}$ respectively). The peptide charges are also significantly different: -17 me for the $\mathrm{P}_{1} \mathrm{P}_{2}$ and 21 me for the $\beta_{1} \beta_{2}$ conformations compared to -2 me and 37 me respectively for the flexible water case (with $\mathrm{PCM}$ the values are 7 me for $\mathrm{P}_{1} \mathrm{P}_{2}$ and 38 me for $\beta_{1} \beta_{2}$ in the case of rigid waters compared to 28 me and 59 me respectively in the 
case of flexible waters.) This level of sensitivity to local interactions highlights the need to develop "micro-fine-grained" force fields ${ }^{4}$ to achieve reliable descriptions of $\mathrm{P} / \beta$ propensity from MD simulations. Structure agreement itself is a necessary but is not a sufficient requirement to guarantee energy accuracy.

Amyloid Formation. There are almost 70 human diseases, both neurodegenerative (such as Alzheimer's and Parkinson's) and non-neuropathic (such as type II diabetes), that are now associated with the formation of insoluble amyloid fibrils ${ }^{15}$. Structural studies by many physical techniques ${ }^{16}$ find that these fibrils, and the many types of aggregates (polymorphs) that are formed from them, are based on a local cross- $\beta$ chain conformation, said to result from the "misfolding" of their peptides. In the case of the Alzheimer's A $\beta$ peptides, these are of about 40 residues, derived from enzymatic cleavage of its amyloid precursor protein, and are biologically functional in their normally soluble state ${ }^{17}$. The formation of the insoluble amyloid plaque is known to be basically independent of the amino acid sequence and is accepted as an inherent property of the polypeptide chain ${ }^{15}$ (it even being speculated that the cross- $\beta$ motif might have been a common ancestor of protein folds ${ }^{18}$ ). Much has been learned about factors influencing amyloid properties, including those such as hydration defects in the cerebrospinal fluid, which can interfere with the normal clearance of $A \beta$ from the brain ${ }^{19}$, 20,21. Ions such as $\mathrm{Zn}^{2+22}$ and $\mathrm{Al}^{3+23}$ enhance amyloid formation while many molecules are found to inhibit the formation of $\mathrm{A} \beta\left(\mathrm{H}_{2} \mathrm{~S}^{24}\right.$, vitamin $\mathrm{B} 12^{25}$, and ascorbic acid ${ }^{26}$ among others). However, the basic and still unanswered question has been what physical property can lead to the formation of an ordered peptide structure from a supposedly 
unordered chain state. Our studies indicate that the fundamental answer lies in the dependence of the $\mathrm{P} / \beta$ propensity in the chain on the specific nature of its environment.

To further evaluate this view, it is important to first understand the detailed structural nature of the initial soluble state. Such peptide chains have generally been described as being in an intrinsically disordered ${ }^{15}$ or random state, especially from CD spectral analyses. Unfortunately, these have been based on an early assignment to the random state $^{27}$ of a CD spectrum that is now recognized as significantly associated with the PPII conformation $^{1,2}$. A CD study of the $\mathrm{A} \beta(12-28)$ fragment $^{28}$ makes this very clear through the temperature dependence of its spectrum (enhanced PPII contribution at lower temperature) and the presence of an isodichroic point ${ }^{29}$ between the PPII spectrum and one consistent with a basically unordered chain ${ }^{30}$. This composite nature of such soluble systems is further supported by $C D$ studies of $A \beta(1-40)^{31}$. Since PPII conformations in adjacent chains cannot effectively hydrogen-bond to each other, their presence in the peptide chain is clearly antagonistic to chain aggregation and thus to the formation of cross- $\beta$ fibrils.

The possibility of amyloid formation then follows from two important results of our ADP and ATP studies on the influence of other species in the aqueous environment. First, since the P/ $\beta$ propensity favors the PPII conformation in a totally aqueous environment, which is obviously not the case in a normal brain, it must be true that certain compositional features of this natural environment still permit this propensity to prevail. Second, since we find that some added species (or even changes in $\mathrm{pH}^{32}$ ) alter the propensity in favor of the $\beta$ conformation and thus can lead to chain aggregation, it is likely that such an addition to the milieu (including seeding by externally introduced 
amyloid $^{33}$ ) constitutes the initiation of amyloid onset. A shift from the present misfolding to a more useful milieufolding paradigm is therefore an important conceptual step in developing a basic understanding of amyloid development.

\section{CONCLUSIONS}

Our previous ab initio studies on the hydrated alanine dipeptide ${ }^{4}$ and our present similar analysis of the comparable alanine tripeptide show that the relative energies of the PPII and $\beta$ conformations, the $\mathrm{P} / \beta$ propensity, depends on the local environment, favoring the $\mathrm{P}$ and $\mathrm{P}_{1} \mathrm{P}_{2}$ conformers in the $\mathrm{ADP}$ and $\mathrm{ATP}$ cases, respectively, in a pure aqueous environment and the $\beta$ and $\beta_{1} \beta_{2}$ conformers in the case of an added $\mathrm{HCl}$ molecule in the system. We believe that other molecules can have the same effect. Since our ab initio approach cannot be used to establish the magnitude of this dependence for longer chains and other side chains, as is desirable, such studies will necessarily have to depend on molecular dynamics simulations. It is quite possible that present force fields are not able to provide a quantitatively reliable description, and we show that this is indeed a problem in the case of an ab initio calculation of ATP with rigid rather than flexible waters. Force fields will need to demonstrate their ability to reproduce such obviously subtle hydrogen-bond-dependent effects.

Implementation of our proposed alternative paradigm of protein milieufolding diseases can lead to better-targeted experimental approaches in studying the onset phase of amyloid formation. We have pointed to the desirability, since neurodegenerative diseases are usually age-related, of monitoring compositional changes in the cerebrospinal fluid that, through lifestyle and/or environmental factors, influence $\mathrm{P} / \mathrm{\beta}$ 
propensity ${ }^{4}$ It may also be the case that blood-brain barrier (BBB) changes, already known to be associated with Alzheimer's ${ }^{34}$, are involved, perhaps through gut microbiota influence on BBB permeability ${ }^{35}$. Detailed knowledge of the initial physical steps in favoring $\beta$ over PPII polypeptide chain conformations most likely holds the keys to enabling effective interventions in the formation of biologically destructive amyloid deposits.

\section{ACKNOWLEDGEMENT}

A major portion of our calculations was made possible by the donation of time on the computational facilities of Charles L. Brooks III. For this we are deeply grateful. 


\section{REFERENCES}

1. Tiffany, M. L.; Krimm, S. New Chain Conformations of Poly(glutamic acid) and Polylysine. Biopolymers 1968, 6, 1379-1381.

2. Krimm, S.; Tiffany, M. L. The Circular Dichroism Spectrum and Structure of Unordered Polypeptides and Proteins. Israel Journal of Chemistry 1974, 12, 189-200.

3. Mirkin, N. G.; Krimm, S. Water Interaction Differences Determine the Relative Energetic Stability of the Polyproline II Conformation of the Alanine Dipeptide in Aqueous Environments. Biopolymers 2012, 97, 789-794.

4. Mirkin, N. G.; Krimm, S. Other Species in the Aqueous Environment of a Peptide Can Invert its Intrinsic Solvated Polyproline II/Beta Propensity: Implications for Amyloid Formation. Biopolymers 2016, 105, 305-311.

5. Gaussian 09, Revision A.2 Frisch, M. J., et al., Gaussian, Wallingford, CT, 2009.

6. Chai, J.; Head-Gordon, M. Long-range Corrected Hybrid Density Functionals with Damped Atom-Atom Dispersion Corrections Phys. Chem. Chem. Phys. 2008, 10, 66156620.

7. van der Vaart, A.; Merz, Jr. M. The Role of Polarization and Charge Transfer in the Solvation of Biomolecules. J. Am. Chem. Soc. 1999, 121, 9182-9190.

8. Dal Peraro, M.; Raugei, S.; Carloni, P.; Klein, M. L. Solute-Solvent Charge Transfer in Aqueous Solution. ChemPhysChem 2005, 6, 1715-1718.

9. Bradbury, E. M.; Brown, L.; Downie, A. R.; Elliott, A.; Fraser, R. D. B.; Hanby, W. E.; McDonald, T. R. R. The "Cross- $\beta$ ” Structure in Polypeptides of Low Molecular Weight. J. Mol. Biol.1960, 2, 276-286. 
10. Astbury, W. T.; Dickinson, S.; Bailey, K. The X-ray Interpretation of Denaturation and the Structure of the Seed Globulins. Biochem. J. 1935, 29, 2351-2360.

11. Astbury, W. T.; Beighton, E.; Parker, K. D. The Cross- $\beta$ Configuration in Supercontracted Proteins. Biochim. Biophys. Acta 1959, 35, 17-25.

12. Palmo, K.; Mannfors, B.; Mirkin,N. G.; Krimm, S. Potential Energy Functions: From Consistent Force Fields to Spectroscopically Determined Polarizable Force Fields. Biopolymers 2003, 68, 383-394.

13. Hagler, A. T. Quantum Derivative Fitting and Biomolecular Force Fields: Functional Form, Coupling Terms, Charge Flux, Nonbond Anharmonicity, and Individual Dihedral Potentials. J. Chem. Theory Comput., 2015, 11, 5555-5572.

14. Meral, D.; Toal, S.; Schweitzer-Stenner, R.; Urbanc, B. Water-Centered Interpretation of Intrinsic PPII Propensities of Amino Acid Residues: In Vitro-Driven Molecular Dynamics Study. J. Phys. Chem. 2015, 119, 13237-13251.

15. Chiti, F.; Dobson, C. M. Protein Misfolding, Amyloid Formation, and Human Disease: A Summary of Progress Over the Last Decade. Annu. Rev. Biochem. 2017, 86, 27-68.

16. Eisenberg, D. S.; Sawaya, M. R. Structural Studies of Amyloid Proteins at the Molecular Level. Annu. Rev. Biochem. 2017, 86, 69-95.

17. Habib, A.; Sawmiller, D.; Tan, J. Restoring Soluble Amyloid Precursor Protein $\alpha$ Functions as a Potential Treatment for Alzheimer's Disease. J. Neuroscience Research 2017, 95, 973-991.

18. Greenwald, J.; Riek, R. On the Possible Amyloid Origin of Protein Folds. J. Mol. Biol. 2012, 421, 417-426. 
19. Mukherjee, S.; Chowdhury, P.; Gai, F. Effect of Dehydration on the Aggregation

Kinetics of Two Amyloid Peptides. J. Phys.Chem. B. 2009, 113, 331-335.

20. Igarashi, H.; Suzuki, Y.; Kwee, I. L.; Nakada, T. Water Influx into Cerebrospinal Fluid is Significantly Reduced in Senile Plaque Bearing Transgenic Mice, Supporting Beta-Amyloid Clearance Hypothesis in Alzheimer's Disease. Neurological Res. 2014, 36, 1094-1098.

21. Simon, M. J.; Illif, J. J. Regulation of Cerebrospinal Fluid (CSF) Flow in Neurodegenerative, Neurovascular and Neuroinflammatory Disease. Biochim. Biophys. Acta 2016, 1862, 442-451.

22. Guo, J.; Yu, L.; Sun, Y.; Dong. X. Kinetic Insights into $\mathrm{Zn}^{2+}$-Induced Amyloid $\beta$ Protein Aggregation Revealed by Stopped-Flow Fluorescence Spectroscopy. J. Phys. Chem. B 2017, 121, 3909-3917.

23. Bondy, S. C. Prolonged Exposure to Low Levels of Aluminum Leads to Changes Associated with Brain Aging and Neurodegeneration. Toxicology 2014, 315, 1-7.

24. Rosario-Alomar, M. F.; Quiñones-Ruiz, T.; Kurouski, D.; Sereda, V.; Ferreira, E. B.; Jesús-Kim, L. D.; Hernández-Rivera, S.; Zagorevski, D. V.; López-Garriga, J.; Lednev, I. K. Hydrogen Sulfide Inhibits Amyloid Formation. J. Phys. Chem. B. 2015, 119, 12651274.

25. Alam, P.; Siddiqi, M. K.; Chaturvedi, S. K.; Zaman, M. Vitamin B12 Offers Neuronal Cell Protection by Inhibiting A $\beta-42$ Amyloid Fibrillation. International Journal of Biological Macromolecules 2017, 99, 477-482.

26. Alam, P.; Beg, A. Z.; Siddiqi, M. K.; Chaturvedi, S. K.; Rajpoot, R. K.; Ajmal, M. R.; Zaman, M.; Abdelhameed, A. S.; Khan, R. H. Ascorbic Acid Inhibits Human Insulin 
Aggregation and Protects Against Amyloid Induced Cytotoxicity. Archives of Biochemistry and Biophysics 2017, 621, 54-62.

27. Greenfield, N.; Fasman, G. D. Computed Circular Dichroism Spectra for the Evaluation of Protein Conformation. Biochemistry 1969, 8, 4108-4116.

28. Jarvet, J.; Damberg, P.; Bodell, K.; Eriksson, L. E. G.; Gräslund, A. Reversible Random Coil to $\beta$-Sheet Transition and the Early Stage of Aggregation of the $A \beta(12-28)$ Fragment from the Alzheimer Peptide. J. Am. Chem. Soc. 2000, 122, 4261-4268.

29. Tiffany, M. L.; Krimm, S. Effect of Temperature on the Circular Dichroism Spectra of Polypeptides in the Extended State. Biopolymers 1972, 11, 2309-2316.

30. Tiffany, M. L.; Krimm, S. Circular Dichroism of the "Random" Polypeptide Chain. Biopolymers 1969, 8, 347-359.

31. Brännström, A.; Õhman, A.; Nilsson, L.; Pihl, M.; Sandblad, L. The N-terminal Region of Amyloid $\beta$ Controls the Aggregation Rate and Fibril Stability at Low pH Through a Gain of Function Mechanism. J. Am. Chem. Soc. 2014, 136, 10956-10964.

32. Kobayashi, S.; Tanaka, Y.; Kiyono, M.; Chino, M.; Chikuma, T.; Hoshi, K.;

Ikeshima, H. Dependence $\mathrm{pH}$ and Proposed Mechanism for Aggregation of Alzheimer's Disease-Related Amyloid-(1-42) Protein J. Mol. Structure. 2015, 1094, 100-117.

33. Walker, L. C.; Schelle, J.; Jucker, M. The Prion-Like Properties of Amyloid- $\beta$ Assemblies: Implications for Alzheimer's Disease. Cold Spring Harb. Perspect. Med. 2016, doi:10.1101/cshperspect.a024398, 1-14.

34. Zenaro, E.; Piacentino, G.; Constantin, G. The Blood-Brain Barrier in Alzheimer's Disease. Neurobiology of Disease. 2017, 107, 41-56. 
35. Braniste, V.; Al-Asmakh, M.; Kowal, C.; Anuar, F.; Abbaspour, A.; Tóth, M.;

Korecka, A.; Bakocevic, N.; Ng, L. G.; Kundu, P. et al. The Gut Microbiota Influences

Blood-Brain Barrier Permeability in Mice. Sci. Transl. Med. 2014, 6 263ra158, 1-11. 
Table 1. Properties of $\operatorname{ATP}\left(\mathrm{H}_{2} \mathrm{O}\right)_{19}$ and $\operatorname{ATP}\left(\mathrm{H}_{2} \mathrm{O}\right)_{19}(\mathrm{HCl})$
System
$\left(\varphi_{1}, \psi_{1}\right) \quad\left(\varphi_{2}, \psi_{2}\right)$
$\Delta \mathrm{E}^{a} \mathrm{q}(\mathrm{pep})^{b}$
$\mathrm{q}\left(\mathrm{Cl}^{-}\right)^{b}$

$\operatorname{ATP}\left(\mathrm{H}_{2} \mathrm{O}\right)_{19}$
$\mathrm{P}_{1} \mathrm{P}_{2}$
$\left(-81^{\circ}, 135^{\circ}\right)\left(-65^{\circ}, 146^{\circ}\right)$
0
$-2$
$\beta_{1} \beta_{2}$
$\left(-158^{\circ}, 150^{\circ}\right)\left(-163^{\circ}, 128^{\circ}\right)$
$-1.80$
37

$\operatorname{ATP}\left(\mathrm{H}_{2} \mathrm{O}\right)_{19} \mathrm{PCM}$
$\mathrm{P}_{1} \mathrm{P}_{2}$
$\left(-83^{\circ}, 138^{\circ}\right)\left(-67^{\circ}, 141^{\circ}\right)$
0
28
$\beta_{1} \beta_{2}$
$\left(-154^{\circ}, 140^{\circ}\right)\left(-153^{\circ}, 121^{\circ}\right)$
$-2.62$
59

$\operatorname{ATP}\left(\mathrm{H}_{2} \mathrm{O}\right)_{19}(\mathrm{HCl})$

$\begin{array}{lrccr}\mathrm{P}_{1} \mathrm{P}_{2} & \left(-92^{\circ}, 115^{\circ}\right)\left(-75^{\circ}, 137^{\circ}\right) & 0 & -30 & -806 \\ \beta_{1} \beta_{2} & \left(-147^{\circ}, 148^{\circ}\right)\left(-146^{\circ}, 116^{\circ}\right) & 2.47 & -6 & -814\end{array}$

$\operatorname{ATP}\left(\mathrm{H}_{2} \mathrm{O}\right)_{19}(\mathrm{HCl})$ PCM

\begin{tabular}{|c|c|c|c|c|}
\hline $\mathrm{P}_{1} \mathrm{P}_{2}$ & $\left(-91^{\circ}, 118^{\circ}\right)\left(-74^{\circ}, 132^{\circ}\right)$ & 0 & -10 & -837 \\
\hline$\beta_{1} \beta_{2}$ & $\left(-146^{\circ}, 144^{\circ}\right)\left(-144^{\circ}, 120^{\circ}\right)$ & 2.86 & 36 & -862 \\
\hline
\end{tabular}

${ }^{a} \Delta \mathrm{E}=\mathrm{E}(\mathrm{PP})-\mathrm{E}(\beta \beta), \mathrm{kcal} / \mathrm{mol}$.

${ }^{b}$ NPA charge in me. 
bioRxiv preprint doi: https://doi.org/10.1101/238006; this version posted December 22, 2017. The copyright holder for this preprint (which was not certified by peer review) is the author/funder. All rights reserved. No reuse allowed without permission. 